\title{
Extensive epidural cufflike growth of malignant pleural mesothelioma causing spinal cord compression
}

Johanna M. M. Gijtenbeek MD, ${ }^{a}$ H. I. Piccardt Brouwer, MD, ${ }^{a}$ Rudolf H. Boerman, MD, PhD,

Ruurd G. Wiggenraad, MD, ${ }^{c}$ Charles J. Vecht, MD, PhD, ${ }^{d}$ and Peter A. E. Sillevis Smitt, MD, PhD,

Nijmegen, Arnhem, The Hague, and Rotterdam, The Netherlands

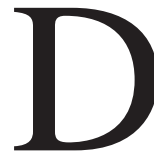

espite multimodal therapy, the prognosis of pleural malignant mesothelioma remains dismal, with a median survival of less than 1 year. ${ }^{1,2}$ The disease usually progresses locally by direct extension into the pulmonary parenchyma, the chest wall, and mediastinum, and most patients ultimately die of respiratory failure. ${ }^{2,3}$ Hematologic spread may occur in the late stages of the disease, but neurologic complications have only rarely been reported. We describe 4 patients with spinal epidural extension of a paravertebral malignant mesothelioma, resulting in signs of spinal cord compression. The neurologic complication developed 4 months and 2, 6, and 7 years after diagnosis, indicating a remarkably indolent course of malignant mesothelioma in most of these patients.

\section{Clinical Summaries}

PATIENT 1. A 56-year-old woman was referred because of progressive low back pain radiating to the back of her legs. She had been treated for pleural effusion for the previous 7 years, and although a pleural tumor was suspected, repeated pleural biopsies were not diagnostic. She had been exposed to asbestos by way of her husband's working clothes. Computed tomographic scan of the lumbar spine showed masses in the left intervertebral foramina at L2-L3 and L3-L4, with thickening of the left psoas muscle. A muscle biopsy specimen revealed malignant mesothelioma. Three months later, the patient had uncontrollable pain and weakness in both legs. A computed tomographic scan of the spine (T7-L5) showed paravertebral masses growing into the intervertebral foramina at all levels. After 4 months, intolerable neck pain, progressive leg weakness, and ataxic gait had developed. Magnetic resonance imaging (MRI) showed epidural tumor growth at the thoracic and cervical levels, extending through the foramen magnum into the skull (Figure 1). One week later, the patient's condition deteriorated, and she died of respiratory failure. At autopsy,

From the Department of Neurology, University Medical Center, Nijmegen, ${ }^{a}$ the Department of Neurology, Rijnstate Hospital, Arnhem, ${ }^{\mathrm{b}}$ the Departments of Radiotherapy ${ }^{\mathrm{c}}$ and Neurology, ${ }^{\mathrm{d}}$ Medical Center Haaglanden, The Hague, and the Department of Neuro-Oncology, Dr Daniel den Hoed Cancer Center/University Hospital, Rotterdam, ${ }^{\mathrm{e}}$ The Netherlands.

Received for publication Dec 14, 2001; accepted for publication Jan 8, 2002

Address for reprints: Johanna M. M. Gijtenbeek, MD, Department of Neurology, University Medical Center Nijmegen, PO Box 9101, 6500 HB Nijmegen, The Netherlands.

J Thorac Cardiovasc Surg 2002;124:200-2

Copyright $(9) 2002$ by The American Association for Thoracic Surgery

$0022-5223 / 2002 \$ 35.00+0 \quad \mathbf{1 2 / 5 4 / 1 2 2 8 6 8}$

doi: $10.1067 / \mathrm{mtc} .2002 .122868$

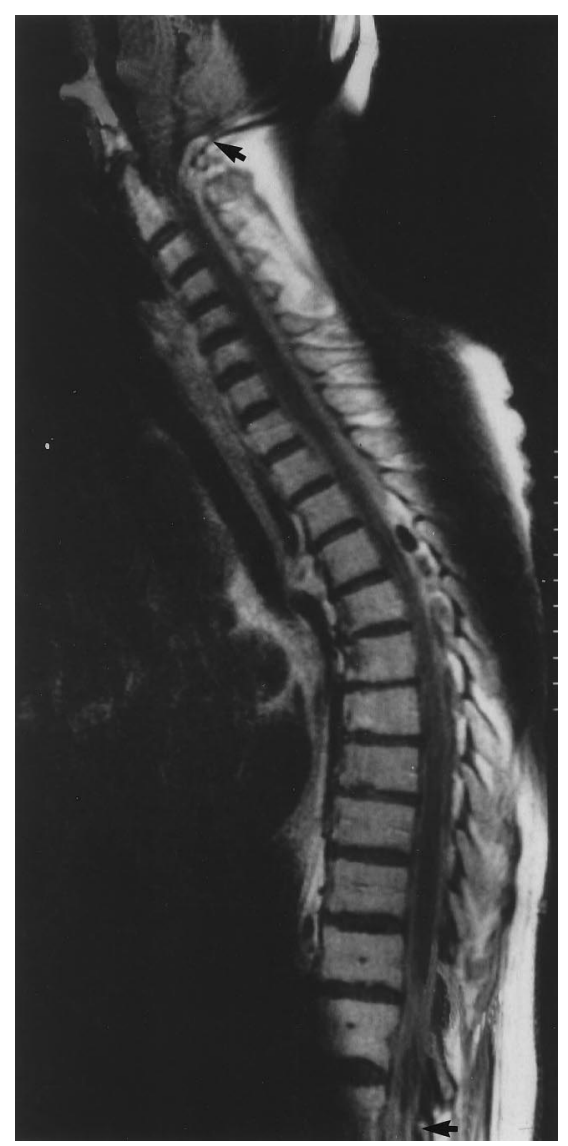

Figure 1. $\mathrm{T}_{1}$-weighted sagittal MRI of patient 1 after administration of gadolinium shows an epidural tumor mass in the cervical and thoracic spinal canal, extending through the foramen magnum into the skull (arrow).

tumor was found in the left thoracic wall, the pericardium, the diaphragm, and the left psoas muscle. At all levels the tumor extended through the intervertebral foramina into the spinal canal. Microscopic examination of the dura at the foramen magnum showed malignant mesothelioma.

PATIENT 2. A 46-year-old man sought treatment with pain in the lumbar spine radiating to the loin. Occasionally he had noticed loss of strength in his right leg, which lasted as long as 15 seconds. Six years previously, malignant mesothelioma of the right pleura had been diagnosed and treated with six courses of cisplatin and irinotecan, followed by focal radiotherapy. The patient had a history of insulin-dependent diabetes for years, and neurologic 


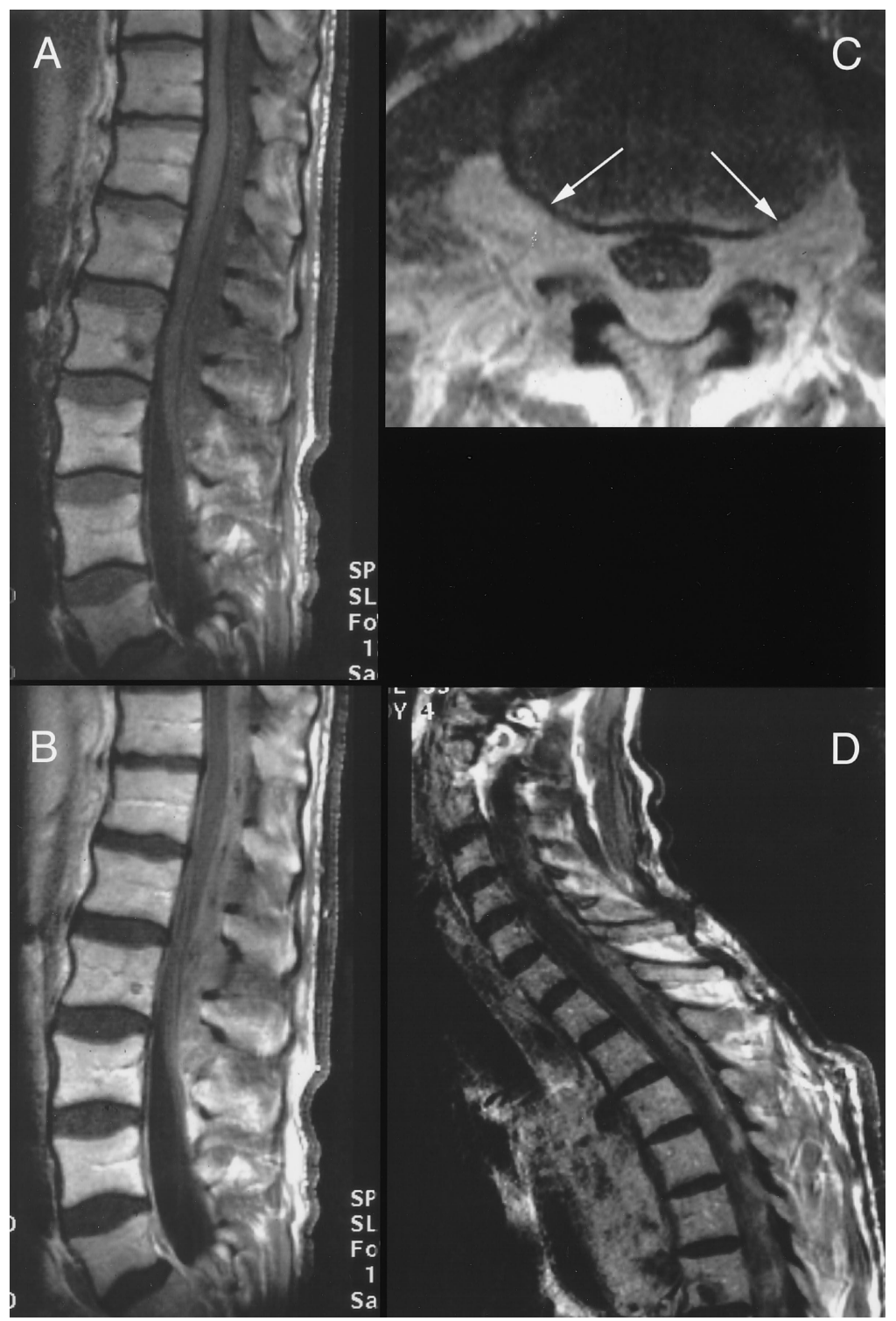

Figure 2. $T_{1}$-weighted sagittal MRI of patient 2 before $(A)$ and after $(B)$ administration of gadolinium demonstrate an epidural mass extending from T10 to $\mathrm{L}_{3} \mathrm{~T}_{1}$-weighted transverse MRI after gadolinium administration (C) shows growth of tumor through the intervertebral foramina (arrows). Five months later, $\mathrm{T}_{1}$-weighted sagittal MRI (D) demonstrates extension of the epidural mass up to the foramen magnum.

examination was compatible with diabetic neuropathy. MRI of the thoracic spine showed growth of the malignant mesothelioma through the intervertebral foramina extending into the epidural space, resulting in an epidural cuff reaching from T10 to L3 (Figure 2, $A-C$ ). The patient was treated with radiotherapy (30 Gy) to T8-L5, with improvement of pain. Five months later, weakness of both legs developed, and the was no longer able to leave his bed. MRI showed extension of the epidural cuff up to the foramen magnum, compressing the spinal cord (Figure 2,D). One month later, the patient died of respiratory failure. Autopsy was not performed.

PATIENT 3. A 41-year-old woman with pleural malignant mesothelioma did not respond to initial treatment with doxorubicin.
Radiotherapy was applied for painful tumor extension into the right breast, with good palliative effect. Two years later, a painful mass developed at the right side of her neck. Cytologic puncture confirmed the diagnosis of malignant mesothelioma. At that time the patient noticed problems walking, and on neurologic examination weakness of both legs and a pin-prick sensory level below C6 was found. MRI showed tumor extension from the right upper thorax into the neck and through the lower cervical foramina into the epidural space, causing cervical spinal cord compression. Palliative radiotherapy ( $37 \mathrm{~Gy})$ to $\mathrm{C} 2-\mathrm{T} 1$ induced a complete regression of the mass in the neck and nearly complete resolution of the weakness. Two months later, the patient had pain in both shoulders and the lower back, with deterioration of muscle strength in both 
legs. MRI showed a cufflike epidural tumor extending from $\mathrm{C} 2$ to the sacrum. Radiotherapy (25 Gy) was given to $\mathrm{C} 1$ and the thoracic and lumbar spine. Neurologic improvement was observed for a period of 3 weeks, after which tumor recurred on cervical and spinal levels. Reirradiation (1 fraction of $8 \mathrm{~Gy}$ ) was instituted at the levels of C5 to T3, but no clinical improvement was observed. Five weeks later, the patient died of respiratory failure. At autopsy, the dura of the spinal cord was surrounded by a thick, yellow, slimy mass, which proved on microscopic examination to be a partially necrotic malignant mesothelioma.

PATIENT 4. A 53-year-old man sought treatment with severe back pain radiating to the right loin and abdomen. Four months earlier, a right pleural malignant mesothelioma that extended retroperitoneally had been diagnosed. The patient had received four cycles of cisplatin and ifosfamide chemotherapy when the back pain occurred. The lower thoracic spine was tender on percussion. Results of the neurologic examination were otherwise normal.

MRI demonstrated a large paravertebral mass invading multiple thoracic vertebrae and intervertebral foramina. At the T10 level, an epidural cuff caused spinal cord compression. The patient was treated with dexamethasone and received $30 \mathrm{~Gy}$ of radiotherapy. Within 1 month of radiotherapy, the patient died of respiratory failure. Autopsy was not performed.

\section{Discussion}

In general, pleural malignant mesothelioma is locally invasive, and progression tends to occur by direct extension of tumor into the pulmonary parenchyma, into the chest wall, into the mediastinum, and through the diaphragm into the abdominal cavity. ${ }^{3}$ Distant metastases, mostly to the lungs, may occur in later stages of the disease. Malignant mesothelioma rarely metastasizes to the nervous system, and only a few cases have been reported of malignant mesothelioma invading the spinal canal. ${ }^{4,5}$

Here we present the cases of 4 patients with pleural malignant mesothelioma and neurologic complications resulting from spinal epidural tumor growth. A remarkable growth pattern consisting of widespread cufflike epidural extension was observed with MRI and confirmed by autopsy in 2 cases. In these patients, the tumor respected anatomic boundaries and spread upward through the epidural space, initially causing root pain and only in later stages causing signs of spinal cord compression. A feature of malignant mesothelioma is its tendency to spread along preexisting tissue planes. The reason for the extensive spinal extension in our patients is unclear, but it may be related to the relatively protracted course of the disease. Although median survival is less than 1 year after onset of symptoms, ${ }^{2} 2$ of the patients survived for more than 6 years.

Malignant mesothelioma can spread to the spinal canal by direct extension through the intervertebral foramina (as in our patients), by hematogenous spread to the spinal meninges,${ }^{4}$ and by perineural growth along a single nerve root. ${ }^{5}$ The patients initially had back pain and radicular pain (arm, leg, loin, or thoracic radiating pain). Radicular pain should alert the clinician to the possibility of epidural tumor in patients with known or suspected malignant mesothelioma. In case of epidural extension, radiotherapy should be immediately instituted to save neurologic function. New symptoms arising along the spinal axis indicate further extension of the tumor and should be promptly evaluated.

\section{References}

1. Edwards JG, Abrams KR, Leverment JN, Spyt TJ, Waller DA, O'Byrne KJ. Prognostic factors for malignant mesothelioma in 142 patients: validation of CALGB and EORTC prognostic scoring systems. Thorax. 2000;55:731-5.

2. Lee YC, Light RW, Musk AW. Management of malignant pleural mesothelioma: a critical review. Curr Opin Pulm Med. 2000;6:267-74.

3. Antman KH. Natural history and epidemiology of malignant mesothelioma. Chest. 1993;103(4 Suppl):373S-6S.

4. Murray JB, Neilly JB, Hadley D, Moran F, McKean M. Diffuse meningeal thickening associated with pleural mesothelioma. Thorax. 1990;45:70-1.

5. Steel TR, Allibone J, Revesz T, D'Arrigo C, Crockard HA. Intradural neurotropic spread of malignant mesothelioma: case report and review of the literature. J Neurosurg. 1998;88:122-5. 